\title{
The Long-Lived Photon Echo Response Locking Effect in the Presence of External Non-Resonant Laser Pulses with a Different Spatial Orientation
}

\author{
Leonid A. Nefed'ev, Elza I. Hakimzyanova, Guzel I. Garnaeva \\ Department of Educational Technologies in Physics, Kazan Federal University, Kazan, Russia \\ Email: nefediev@yandex.ru, Elzahakim@yandex.ru,guzka-1@yandex.ru
}

Received September 9, 2013; revised October 7, 2013; accepted October 28, 2013

Copyright (C) 2013 Leonid A. Nefed'ev et al. This is an open access article distributed under the Creative Commons Attribution License, which permits unrestricted use, distribution, and reproduction in any medium, provided the original work is properly cited.

\begin{abstract}
The correlation of the inhomogeneous broadening of the resonance transition at different time intervals and the efficiency of long-lived photon echo response locking by the action of standing waves of non-resonant laser pulses are considered. It is shown that the long-lived photon echo response locking effect may be observed even at the angles of the relative orientation of the non-resonant standing wave laser pulses of less than one degree, due to the change in the correlation coefficient of inhomogeneous broadening at time intervals between the first and the second and after the third resonant laser pulses.
\end{abstract}

Keywords: Photon Echo; The Information Locking; Spatial Non Uniform Electromagnetic Fields; Relaxation Processes; Time of The Response; External Space-Non Uniform Non Resonant Laser Pulses

\section{Introduction}

At present, a problem of erasing information is the greatest technical challenges in creating specific patterns of optical storage devices. To solve this problem, various methods are proposed [1-4]. They are based on the elimination of the space-frequency modulation of the resonance levels population by acting on the system with defined sequence of optical pulses. However, all offered schemes of removal of the information are difficult enough for their technical embodiment. In addition, the process of erasing of the information is energetically unprofitable, since its implementation requires the energy of the same order as that for recording information. From this perspective, the information locking is more profitable, which means creating the conditions under which the information doesn't show up in the form of the optical response of a resonant medium. This can be achieved by breaking the time-frequency correlation of inhomogeneous broadening of the resonance line at different time intervals [5].

It is known that the formation of photon echo response consists of two main stages: phase mismatching of the oscillating dipole moments of optical centers and their subsequent phase matching. At the second stage, the medium becomes macroscopically polarized, which is observed as a specific response. Thus, an insignificant violation of the rigid time-frequency correlation of inhomogeneous broadening should lead to a significant reduction in the response intensity (the reversible destruction of the phase memory of a resonance medium with a possibility of its reconstruction). This effect can be obtained by subjecting a resonant medium to the action of different spatially inhomogeneous external perturbations at different time intervals. Such an action should lead to random shifts or splitting of the initial monochromatic components of the inhomogeneously broadened line.

We should note that the effect of locking of long-lived photon echo in a La $\mathrm{F}_{3} \mathrm{Pr}^{3+}$ crystal is theoretically predicted and experimentally confirmed in [6].

A similar effect can be achieved through non-resonant interaction of a quantum system with laser pulses. If the non-resonant laser fields have spatial inhomogeneity, the energy shifts $\delta \mathrm{E}$ are functions of the coordinates $\delta \mathrm{E}(\boldsymbol{r})$, which leads to additional inhomogeneous broadening of the resonance transition in the sample.

In this study we consider the correlation of the inhomogeneous broadening of the resonance transition at different time intervals and the efficiency of long-lived photon echo response locking by the action of standing waves of non-resonant laser pulses. 


\section{The Basic Equations}

Let us consider the formation of a long-lived photon echo under the action of sequence of three resonant laser pulses and state waves of non-resonant laser pulses with a different spatial orientation (Figure 1).

The equation for the single-particle density matrix in a rotating coordinate system can be written as

$$
\partial \tilde{\rho} / \partial t=i / \hbar[B, \tilde{\rho}]
$$

where

$$
\begin{gathered}
B=\tilde{H}_{0 m}+\tilde{U}-\hbar A, \\
\tilde{H}_{0 m}=\mathrm{e}^{i A t} H_{0 m} \mathrm{e}^{-i A t}, \\
\tilde{U}=\mathrm{e}^{i A t} U \mathrm{e}^{-i A t},
\end{gathered}
$$

where $A$ is the matrix of transition to the rotating coordinate system, $U$ is the operator of the resonant interaction with the exciting laser pulses, $H_{0 m}$ is the Hamiltonian of an atom in the external spatially non uniform non resonante laser radiation at $\tau_{m}$-th time interval, $\boldsymbol{r}$ is the radius vector of the atom location. In the case of two-level system, $A=P_{22} \omega_{12}$,

$$
\begin{gathered}
\mathrm{e}^{ \pm i A t}=P_{11}+P_{22} \mathrm{e}^{ \pm i \omega_{12} t}, \\
\tilde{H}_{0 m}=\hbar\left(\Delta+\varepsilon\left(\tau_{m}, \boldsymbol{r}\right)\right), \\
\Delta=\omega_{12}-\omega, \\
\tilde{U}=P_{12} U_{12} \mathrm{e}^{-i \omega_{12} t}+P_{21} U_{21} \mathrm{e}^{i \omega_{12} t}, \\
U_{12}=\frac{1}{2} d_{12} E_{0} \mathrm{e}^{i \omega t-i k r}, \\
U_{21}=\frac{1}{2} d_{21} E_{0} \mathrm{e}^{-i \omega t+i k r},
\end{gathered}
$$

where $P_{i j}$ are the projective matrices (their ij-th element is equal to unity and the other elements are zero), $\omega_{12}$ is the frequency of the resonance transition, $d_{i j}$ is the dipole moment of the resonance transition, $E_{0}$ is the electric field strength of resonant laser pulse and $\boldsymbol{k}$ its wave vector, $\varepsilon\left(\tau_{m}, \boldsymbol{r}\right)$ is an additional frequency shift of the

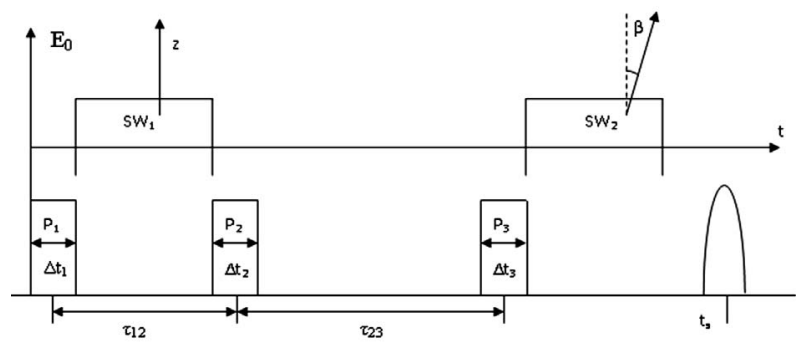

Figure 1. Excitation pulses order in the formation of signals long-lived photon echo $P_{1}, P_{2}$ and $P_{3}$ are the exciting pulses, $\tau_{\mathrm{mn}}$ is a time interval between the $\mathrm{m}$-th and the $\mathrm{n}$-th pulses, $\mathrm{SW}_{1}$ and $\mathrm{SW}_{2}$ are a standing waves, $\tau_{1}$ and $\tau_{2}$ are durations of the non-resonant laser pulses. resonant transition of an atom in a time interval $\tau_{m}$ by external non-resonant spatially inhomogeneous laser radiation.

The dependence $\varepsilon$ from the location of the optical center in the sample is related to the spatial inhomogeneity of nonresonant laser radiation. Such inhomogeneity occurs, for example, under the influence of a standing wave. In this case the energy shift of the n-th state of the atom can be written as $[7,8]$.

$$
\delta E_{n}=\frac{1}{2 \hbar} \sum_{s} \frac{\omega_{n s}\left|d_{n s}\right|^{2}}{\omega_{n s}^{2}-\omega^{2}} E_{0 \eta}^{2} \cos \left(\boldsymbol{k}_{\eta} \boldsymbol{r}_{j}\right)
$$

and

$$
\varepsilon\left(\tau_{\eta}, \boldsymbol{r}\right)=\hbar^{-1}\left(\delta E_{2}(\boldsymbol{r})-\delta E_{1}(\boldsymbol{r})\right)=C_{D} E_{0 \eta}^{2} \cos \left(\boldsymbol{k}_{\eta} \boldsymbol{r}_{j}\right),
$$

where the $C_{D}$ is the constant of the dynamic Stark effect, $E_{0 \eta}$ is the electric field amplitude of the $\eta$-th non-resonant laser pulse, $\omega_{n s}$ is the frequency of transitions between bound states of $\mathrm{n}$ and $\mathrm{s}$. If $\omega \ll \omega_{n s}$ expression (2) becomes the common formula for the quadratic Stark effect

$$
\begin{aligned}
\delta E_{n}(\boldsymbol{r}) & =\frac{1}{2 \hbar} \sum_{s} \frac{\left|d_{n s}\right|^{2}}{\omega_{n s}} E_{0 \eta}^{2} \cos \left(\boldsymbol{k}_{\eta} \boldsymbol{r}_{j}\right) \\
& =\hbar C_{s h} E_{0 \eta}^{2} \cos \left(\boldsymbol{k}_{\eta} \boldsymbol{r}_{j}\right),
\end{aligned}
$$

where $C_{s h}$ is the constant of the quadratic Stark effect. In most cases the constants of quadratic Stark effect for different atomic transitions lie in the range of 10 $\mathrm{Hz} /(\mathrm{B} / \mathrm{cm})^{2}<\mathrm{C}_{\mathrm{sh}}<1 \mathrm{kHz} /(\mathrm{B} / \mathrm{cm})^{2}$ [9], which is comparable with the dynamic Stark effect constants values $C_{D}$.

This allows us to obtain a sufficiently large frequency shifts $\varepsilon\left(\tau_{\eta}, r\right)$ compared to the width of the excitation $k \sigma(k<1)$ of an inhomogeneously broadened line of the resonance transition by selecting the appropriate power non-resonant laser pulses. If the non-resonant frequency $\omega$ of the laser radiation is close to one of the frequencies $\omega_{n s}$ (transitions to the perturbing levels), the values of constant $C_{D}$ can greatly exceed the value of $C_{\mathrm{sh}}$.

The initial distribution of the optical centers over the frequencies $g(\Delta)$ we will assume as Gaussian with dispersion $\sigma^{2}$ :

$$
g(\Delta)=\frac{1}{\sigma \sqrt{2 \pi}} \exp \left[\left(\frac{\Delta}{\sigma \sqrt{2}}\right)^{2}\right]
$$

Comparing the frequencies shifts of optical centers on different time intervals $\tau_{\eta}$, by interaction with a different spatially oriented standing waves it is convenient to define the vector $\boldsymbol{k}_{\eta}$ in the coordinate system $\left(x_{\eta}, y_{\eta}, z_{\eta}\right)$ associated with the direction of propagation of laser radiation:

$$
\boldsymbol{k}_{\eta}=\boldsymbol{i}_{\eta} k_{x_{\eta}}+\boldsymbol{j}_{\eta} k_{y_{\eta}}+\boldsymbol{k}_{\eta} k_{z_{\eta}}
$$


where the $\left(\boldsymbol{i}_{\eta}, \boldsymbol{j}_{\eta}, \boldsymbol{k}_{\eta}\right)$ are the unit vectors of the coordinate system $\left(x_{\eta}, y_{\eta}, z_{\eta}\right)$. In the laboratory coordinate system $(x, y, z)$

$$
\left(\begin{array}{l}
k_{x} \\
k_{y} \\
k_{z}
\end{array}\right)=A\left(\alpha_{\eta}, \beta_{\eta}, \gamma_{\eta}\right)\left(\begin{array}{l}
k_{x_{\eta}} \\
k_{y_{\eta}} \\
k_{z_{\eta}}
\end{array}\right)
$$

where $A$ is the matrix of rotations. From (7) we find that

$$
\begin{aligned}
k_{x}= & k_{x_{\eta}}\left(\cos \alpha_{\eta} \cos \beta_{\eta} \cos \gamma_{\eta}-\sin \alpha_{\eta} \sin \gamma_{\eta}\right) \\
& -k_{y_{\eta}}\left(\cos \alpha_{\eta} \cos \beta_{\eta} \sin \gamma_{\eta}+\sin \alpha_{\eta} \cos \gamma_{\eta}\right) \\
& +k_{z_{\eta}} \cos \alpha_{\eta} \sin \beta_{\eta}, \\
k_{y} & =k_{x_{\eta}}\left(\sin \alpha_{\eta} \cos \beta_{\eta} \cos \gamma_{\eta}+\cos \alpha_{\eta} \sin \gamma_{\eta}\right) \\
& +k_{y_{\eta}}\left(-\sin \alpha_{\eta} \cos \beta_{\eta} \sin \gamma_{\eta}+\cos \alpha_{\eta} \cos \gamma_{\eta}\right) \\
& +k_{z_{\eta}} \sin \alpha_{\eta} \sin \beta_{\eta}, \\
k_{z}=- & k_{x_{\eta}} \sin \beta_{\eta} \cos \gamma_{\eta}+k_{y_{\eta}} \sin \beta_{\eta} \sin \gamma_{\eta}+k_{z_{\eta}} \cos \beta_{\eta} .
\end{aligned}
$$

here $\alpha_{\eta}, \beta_{\eta}$ and $\gamma_{\eta}$ are Euler angles defining the relative orientation by coordinate systems $(x, y, z)$ and $\left(x_{\eta}, y_{\eta}, z_{\eta}\right)$.

\section{The Correlation of Inhomogeneous Broadening and Effectiveness of Locking a Long-Lived Photon Echo Depending on the Relative Orientation of Non-Resonant Laser Pulses}

The coefficient of time-frequency correlation of inhomogeneous broadening at the time intervals $\tau_{\eta}$ and $\tau_{\varphi}$ taking into account (1) and (3) has the form [10]:

$$
\begin{aligned}
& R_{\eta, \varphi} \\
& =\frac{1}{V} \int_{-\infty}^{\infty} \int_{V} \frac{\left[f\left(\tau_{\eta}, \Delta, \boldsymbol{r}\right)-\bar{u}_{\eta}\right]\left[f\left(\tau_{\varphi}, \Delta, \boldsymbol{r}\right)-\bar{u}_{\varphi}\right]}{\sigma_{\eta} \sigma_{\varphi}} g(\Delta) \mathrm{d} V \mathrm{~d} \Delta
\end{aligned}
$$

where $V$ is the volume of the excited part of sample,

$$
\begin{array}{r}
\bar{u}=\frac{1}{V} \int_{-\infty}^{\infty} \int_{V} f(\tau, \Delta, \mathbf{r}) g(\Delta) \mathrm{d} V \mathrm{~d} \Delta, \\
\sigma^{2}=\frac{1}{V} \int_{-\infty}^{\infty} \int_{V}(f(\tau, \Delta, \mathbf{r})-\bar{u})^{2} g(\Delta) \mathrm{d} V \mathrm{~d} \Delta .
\end{array}
$$

The numerical calculation of the correlation coefficient (8), depending on the relative orientation of the non-resonant standing wave of laser pulses is shown in Figure 2. It implies that small changes of the angle between the non-resonant standing waves of laser pulses $\mathrm{CW}_{1}$ and $\mathrm{CW}_{2}$ (within 0.1 degrees) results a change in the correlation coefficient of inhomogeneous broadening at different time intervals that means the destruction of the reversible phase memory of the system.

Figure 3 shows the results of numerical calculation of the intensity of a long-lived photon echo response as a function of angle $\beta$. Analysis of the resulting angular dependence shows that there is effect of locking a long-lived photon echo that takes place if $\beta<0.1$ degrees. Thus, using the excitation scheme of Figure 1, you can create a large number of independent channels of recording and reproducing information, with the angle $\beta$ as associative key of access.

\section{Conclusion}

The formation of the stimulated photon echo in the presence of external non-resonant laser pulses leads to the effect of a long-lived photon echo locking. The informa-

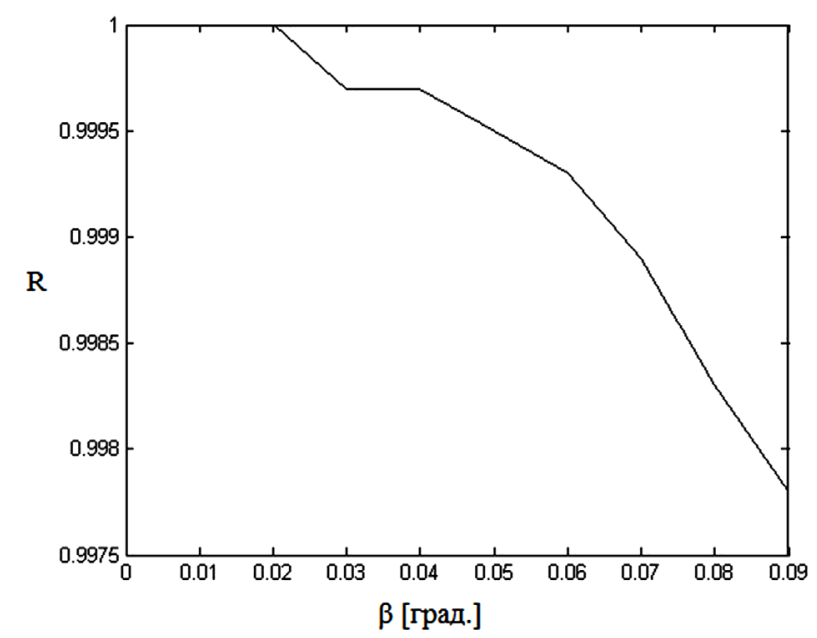

Figure 2. Angular dependence of the frequency-time correlation coefficient in the presence of non-resonant standing waves in $\mathrm{La} \mathrm{F}_{3} \operatorname{Pr}^{3+}, \tau_{1}=\tau_{2}=20 \mathrm{nc}, \sigma=5 \mathrm{nc}^{-1}, \Delta \mathrm{t}_{1}=\Delta \mathrm{t}_{1}=5 \mathrm{nc}$.

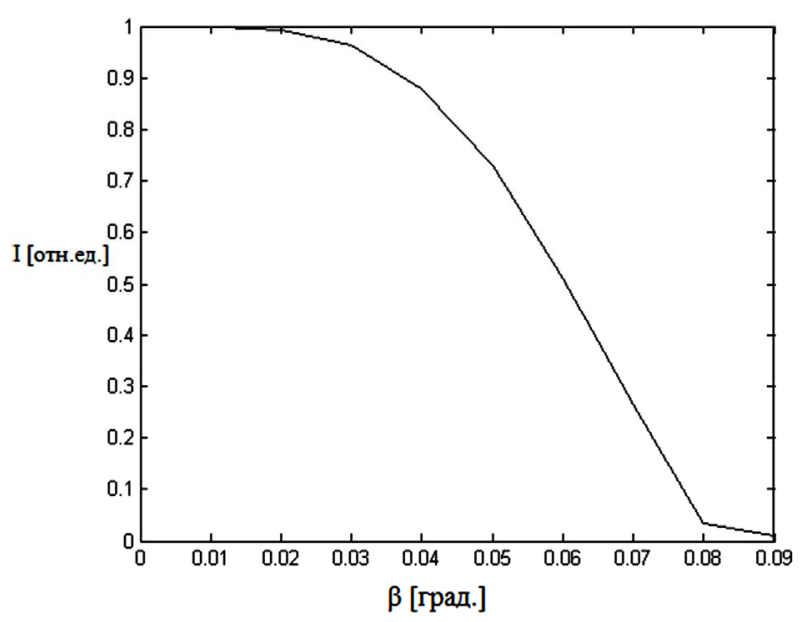

Figure 3. The angular dependence of the long-lived photon echo intensity in the presence of non-resonant standing waves in $\mathrm{La} \mathrm{F}_{3} \mathrm{Pr}^{3+}, \tau_{1}=\tau_{2}=20 \mathrm{nc}, \sigma=5 \mathrm{nc}^{-1}, \Delta \mathrm{t}_{1}=\Delta \mathrm{t}_{1}=$ 5 nc. 
tion locking effect in the presence of non-resonant standing waves occurs if angles of relative orientation of the non-resonant standing waves of laser pulses are less than 0.1 degress.

\section{REFERENCES}

[1] N. N. Akhmediev and B. S. Borisov, "The Prospects of the Effect of Photon Echo in Modern Electronics," Microelectronics, Vol. 15, No. 1, 1986, pp. 25-30.

[2] N. N. Akhmediev and I. V Mel'nikov, "Multiple Stimulated Light Echo in Athree-Tier Environments," Quantum Electric, Vol. 15, No. 12, 1988, pp. 2522-2524.

[3] S. A. Moiseev and E. I. Shtyrkov, "Generation of Transient Inversion Gratings Ultra Period in Media with Phase Memory in the Multi-Pulse Interaction," Quantum Electric, Vol. 18, No. 4, 1991, pp. 447-451.

[4] S. B. Karamyshev, "Theoretical Study of Decay of Frequency-Space Modulation of Level Populations," Laser Physics, Vol. 3, No. 5, 1993, pp. 1037-1041.

[5] A. A. Kalachev, L. A. Nefed'ev and V. V. Samartsev. “'Locking' Echoholographic Information in the Mode of
Photon Echo in Doped Crystals," Optics and Spectroscopy, Vol. 84. No. 5, 1998, pp. 731-734.

[6] V. V. Samartsev, V. A. Zuikov and L. A. Nefed'ev, "Optical Memory Based on a Long-Lived Photon Echo," Journal of Applied Spectroscopy, Vol. 59. No. 5-6, 1993, pp. 766-791. http://dx.doi.org/10.1007/BF00664916

[7] N. B. Delone and V. P. Krainov, "An Atom in a Strong Laser Field," Moscow, 1978, p. 286.

[8] I. I. Sobel'man, "Introduction to the Theory of Atomic Spectra," Science, Moscow, 1967, p. 319.

[9] A. M. Bonch-Bruevich, "Travel Modern Methods of Investigating the Stark Effect in Atoms," Physics-Uspekhi (Advances in Physical Sciences), Vol. 93, No. 1, 1967, pp. 71-110.

[10] L. A. Nefediev and G. I. Khakimzyanova, "The Correlation of Inhomogeneous Broadening and the Efficiency of Data Locking in Optical Echo Processors," Optics and Spectroscopy, Vol. 98, No. 1, 2005, pp. 35-39. http://dx.doi.org/10.1134/1.1858037 